\title{
Congratulation message to Dr. Ali Khademhosseini
}

Published online: 13 January 2012

(C) Springer Science+Business Media, LLC 2012

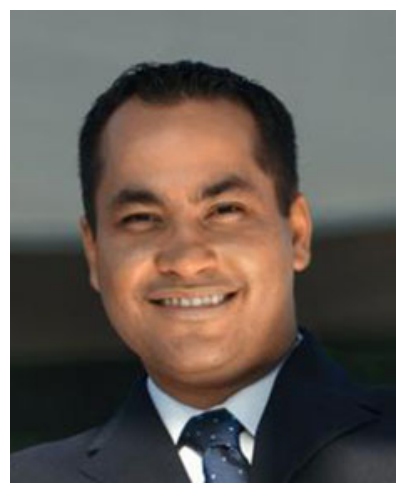

Biomedical Microdevices congratulates Dr. Ali Khademhosseini, for his recent recognition by the U.S. government with the 2011 Presidential Early Career Award for Scientists and Engineers. Dr Khademhosseini is an internationally acclaimed bioengineering and material science researcher, and has earned this recognition for his ground breaking research in tissue engineering systems. We are deeply honored to have Dr Khademhosseini as part of our scientific family, serving as Associate Editor for Biomedical Microdevices, and we thank him for his visionary scientific leadership.

Dr. Khademhosseini gained early recognition for his work with former MIT postdoc Javier Gomez Fernandez, to create a tissue engineering system now known as 'micromasonry' or 'biological Legos'. The system uses a novel biogel that can be used to coat cells and then hardens to create encapsulated cellular building blocks when exposed to light. The blocks are then stacked on a silicon-based polymer scaffold to create a $3 \mathrm{D}$ structure that mimics the tissue microarchitecture that is necessary to create organ ultrastructures like blood vessels. The final structure can be fused by addition of more light-activated bio-gel. This system has been used to successfully to create engineered capillaries. He is currently using this system to create artificial muscles from fish cells for applications in drug screening and robotic devices.

His research team is also using webcam image sensors to monitor the beating of cardiomyocytes, which can monitor the cardiotoxicity commonly caused by medicines. Cardiotoxicity is the main reason for the failure of FDA approval for more than $30 \%$ of pharmaceutical drugs.

Dr. Khademhosseini has significantly advanced the fields of material science and tissue engineering is a relatively short time. He received his Ph.D. in Bioengineering from MIT in 2005, and accepted an appointment to Associate Professor at Harvard-MIT's Division of Health Sciences and Technology (HST), Brigham and Women's Hospital (BWH) and Harvard Medical School (HMS). He also directs a satellite lab at Japan's World Premier International-Advanced Institute for Materials Research (WPI-AIMR) at Tohoku University and is a Harrington fellow in the Biomedical Engineering Department of the University of Texas at Austin.

In his short tenure, he has already has published more than 180 articles which have been cited more than 5900 times. He is currently the second most-cited U.S. bioengineering faculty member in the last five years. He was also named one of the top young innovators under age 35 by Technology Review in 2007.

Other notable accolades for his work include the IUPAC Award (2012), the Pioneers in Miniaturization Prize from the Royal Society for Chemistry (2011), the IEEE Early Career Award in Nanotechnology, the Society for Biomaterials Young Investigator Award (2011), the Office of Naval Research young investigator award (2010), the NSF Career Award (2009), and the American Chemical Society Viktor K. LaMer award (2008). 